\title{
A Translation of UML Components into Formal Specifications
}

\author{
Peng Liang ${ }^{1}$, Annya Romanczuk ${ }^{1}$, and Jean-Claude Royer ${ }^{2}$ \\ ${ }^{1}$ Groupe Objets, Composants, Modèles, Ecole des Mines de Nantes, 4, rue Alfred Kastler - BP \\ 20722, F-44307 Nantes Cedex 3 \\ Liang.Peng@eleve.emn.fr, Annya.Romanczuk@emn.fr \\ Equipe Génie Logiciel, Méthodes et Spécifications Formelles, IRIN - Université de Nantes, 2, \\ rue de la Houssinière, BP 92208, F-44322 Nantes Cedex 03 \\ Jean-Claude.Royer@irin.univ-nantes.fr
}

\begin{abstract}
UML demonstrated that it is a well-suited approach to analyse and design complex systems such as industrial ones. To improve software reliability and reusability the use of formal specification is necessary but it may be difficult. There is a general lack in the formal specification of the UML concepts. This decreases the ability to develop tools and guidelines to help the specifier. Software tools are needed to assist the formal specification process, but also to prove or to verify some parts. In this paper we propose a method to formally specify concurrent and communicating components with data in UML. The principle is, first to complete the design with axioms, second to translate the diagrams into algebraic specifications of data types thanks to our translation tool. We describe the translation principles which are based on previous work around symbolic transition systems and algebraic specifications. Finally, we sketch the implementation of our translation tool using XMI files and the XML4J parser.
\end{abstract}

\section{Introduction}

Object oriented analysis and design languages are very popular in the industrial developer community. In this field, the Unified Modelling Language (UML) [1] is becoming a de facto standard. Using UML, one may represent most of the current applications for software systems. To improve software reliability and reusability the use of formal specification is necessary. It is currently recognised that the UML language suffers from a lack of formal semantics $[2,3]$. This decreases the ability to develop tools and guidelines to help the specifier. Tools are helpful to assist the formal specification process, but also to prove or to verify the resulting software. Designers require a more formal semantics to be able to check, to validate and to refine UML models.

This paper presents an approach to translate some UML models into formal specifications then verification tools may be used to early validate the models. This paper deals only with static classes, active classes, statecharts and collaboration diagrams. There are several important difficulties to provide a formal approach for UML. The first is the need to complete UML diagrams with formal notations to get formal UML components. The second is to give a semantics to the UML diagrams and last is to verify 
or prove properties. Our approach reuses previous experiences with the LOTOS language [4], symbolic transition systems, algebraic specifications [5] and formal software component [6] in order to complete UML diagrams. Our aim is to suggest a method, to propose algorithms and tools to help the specifier. We translate the completed UML diagrams into an homogeneous algebraic model. It allows us to use prover tools like the Larch Prover [7] or PVS [8] for example. Of course we do not address all these problems in this current paper, complementary references are $[9,10,5,11,12]$. We will restrict our study to the translation of UML sequential and concurrent components with data into formal specifications.

The paper is organised as follows. Section 2 is an introduction to the formal background and notations used to complete and to translate UML models. We present our example in Section 3 and assumptions to complete the diagrams. The translation rules for a sequential component are detailed in Section 4, and the concurrent case is described in Section 5. Section 6 overviews the tool we are implementing to support such translation. In Section 7 we compare our approach to other works, then we conclude.

\section{Formal Specification Background}

There are several reasons to use algebraic specifications while many other work rather uses model-oriented specification like Z, B, or VDM, see [13] for example. First, this concept is linked to class. Earlier B. Meyer felt that Abstract Data Types (ADT) are important in the context of object oriented programming (Chapter 6 of [14]). However some difficulties remain about inheritance and concurrency. Second, for more than twenty years, there has been a great amount of work about algebraic specifications and the definition of abstract data types. There are several efficient tools to handle them like the Larch Prover, PVS, or Isabelle. One last interest is that this approach is able to catch both data types and concurrent systems in an homogeneous framework, see [15] for a good survey. We refer here to $[16,15]$ which are rather comprehensive documents about these formal specification techniques. In brief, an algebraic specification of a data type is composed of three main parts:

- A heading part containing informations about the module. Mainly they are: the name (or sort) of the defined data type and the imported modules.

- The signature part describes the operation syntax.

- The axiom part describes the operation semantics.

An observer is an operation whose resulting type is not the defined sort. An internal operation has the defined sort as resulting type. Generators are internal operations which are sufficient to generate all the values of the data type. Since we have partial operations we must cope with the definedness of terms. This is done by a special predicate, the definedness predicate related to the so-called notion of operation precondition. The Figure 1 presents a simple example to illustrate our presentation. The following notations are used in this paper: $\sim$ is logical not, $\bigwedge$ is and, $\backslash$ is or, $=>$ is implication and $=$ is syntactic equality. An axiom like: exists (newStaticBank ( $1 c, p, n)$ ) = has $\left(l_{c}, n\right)$ means that to know if an account number exists it is sufficient to search it in the list of account $l_{\mathrm{C}}$ of the bank. Note that we use positive conditional axiom (or 
Horn clauses) because it gives a framework with good expressiveness and properties. The meaning of an axiom like: exists (newStaticBank (lc, p, n)) => theAccount (newStaticBank $\left(l_{c}, p, n\right)$ ) $=$ find $\left(l_{c}, n\right.$ ) is: IF the account number exists THEN the account object will be the result of a $\mathrm{find}$ operation in the list of accounts. Because such a writing is a bit difficult for a non specialist our method [5] proposes to help the specifier with an automation of the main part. To make reading easier for non specialist such axioms may be rewritten in a more familiar and object oriented way, but we ignore this here.

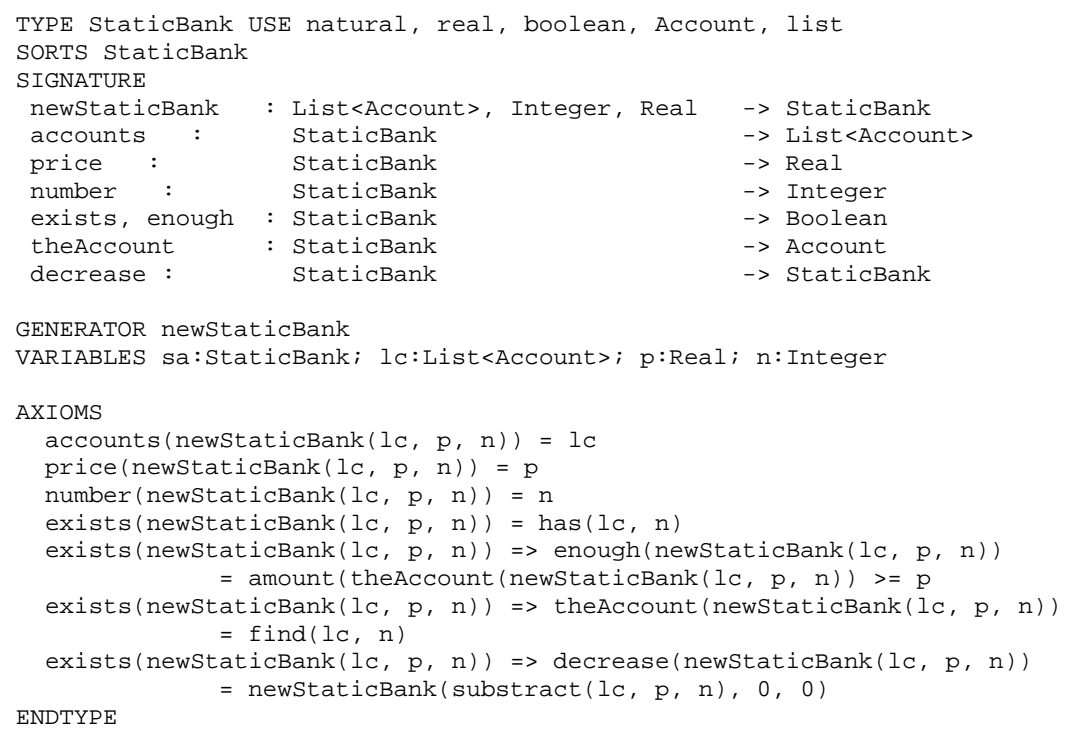

Fig. 1. A Simple Algebraic Specification

This kind of specification describes a data type but it is also possible to specify concurrency and synchronisation [15]. Here we used a proper approach based on Graphic Abstract Data Type [5]. A component is described by a Symbolic Transition System (a STS is a simple form of statechart) and from that an algebraic specification may be built. The semantics of concurrency and synchronisation are obtained from the synchronous product of STSs in a similar way than for the synchronous product of automata [17]. First, we built the free product of the two symbolic transition systems. Second we get out the pair of transitions which are not allowed by the synchronisations. Last, the synchronisations are enriched by communications. An algebraic specification is eventually built from the computed symbolic system. Thus both synchronisation and communication are integrated in an algebraic style.

Once we have completed the UML diagrams the generation of the algebraic specification is completely automatic. This is of course a great advantage from a specifier 
point of view, and also different algebraic languages may be targeted. The approach presented here has two steps:

1. To translate sequential components into algebraic specifications.

2. To built synchronous product of statecharts and to compute algebraic specifications for concurrent components.

\section{Example and Assumptions}

We model a simple example with two concurrent components in UML. This is a part of a seat reservation to illustrate the translation principles. There is a component for the seat reservation and another one for simulating the bank. The client gives its account number when he requests a seat, if there is a seat, the seat reservation invoices the price to the bank. If all is ok the client gets a ticket else the reservation fails.

\subsection{The Bank Component}

We suggest to specify the bank component in the manner described by the Figure 2 . This is a sequential process with an inner static part. It describes the active class, the

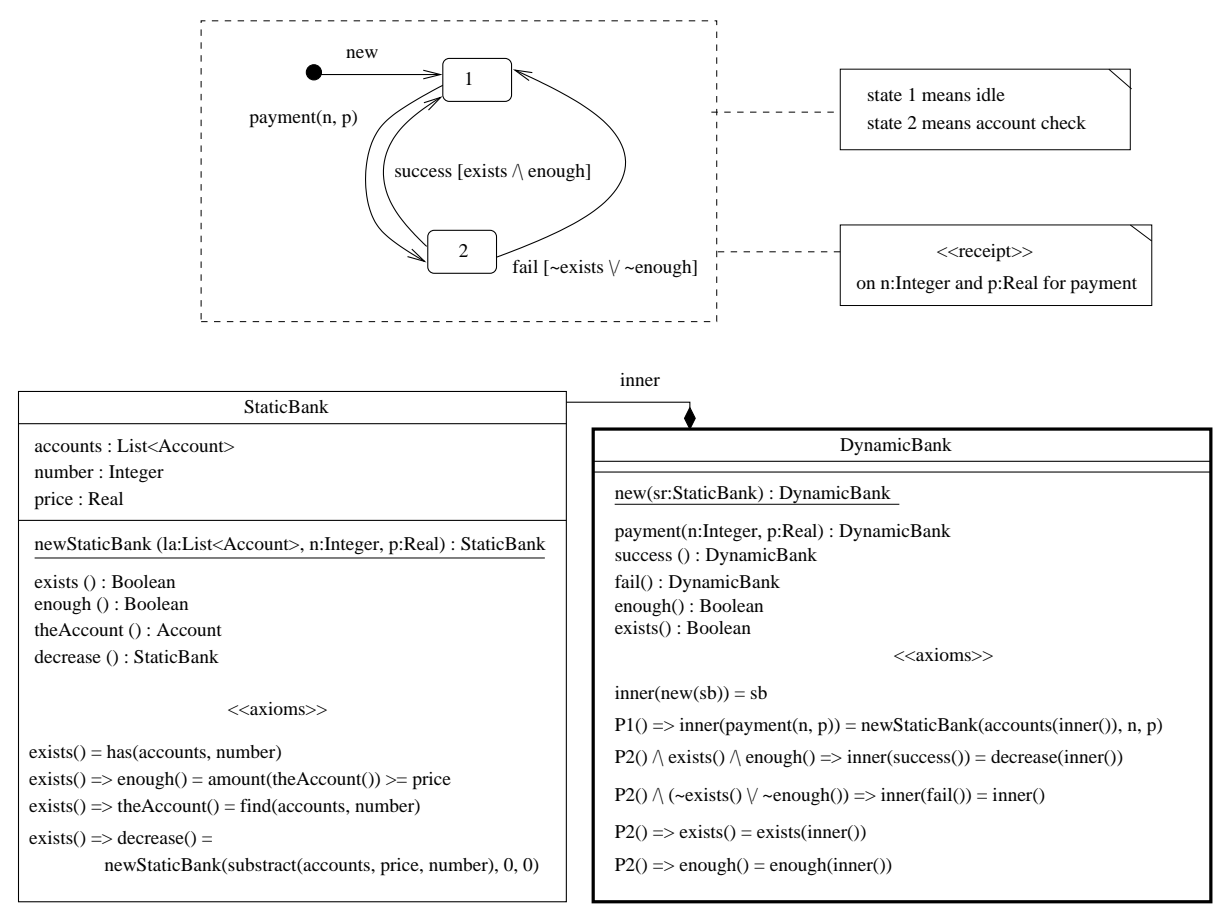

Fig. 2. The Bank Component 
static class and a simple dynamic behaviour. We assume a strong link among the three parts. The main idea is: the dynamic behaviour described by the statechart is an abstract interpretation of the partial abstract data type associated to the class specification. A static class is associated to the active class, it describes the internal state and the private functional behaviour of the component. Thus operations occurring in the statechart correspond to operations of the active class interface. An operation of the active class may call or delegate some computations to the associated static part. We allow the specifier to add axioms to formally describe the operation semantics. This part is introduced by the axioms stereotype. In these axioms P suffixed by a number is the state predicate associated to the number state. The statechart shows three main events and two states. The active class DynamicBank describes what happens when events are triggered. For example if event payment $(n, p)$ occurs in state 1 then the inner part, which represents the data of the bank, memorises the account number $n$ and the price $p$. One may note that if the fail event arises when the system is in state 2 and if the conditions exists or enough are false then nothing is done on the data part of the bank. But if success occurs then the account in the data part will decrease.

The seat reservation component has a similar specification described in Figure 3.

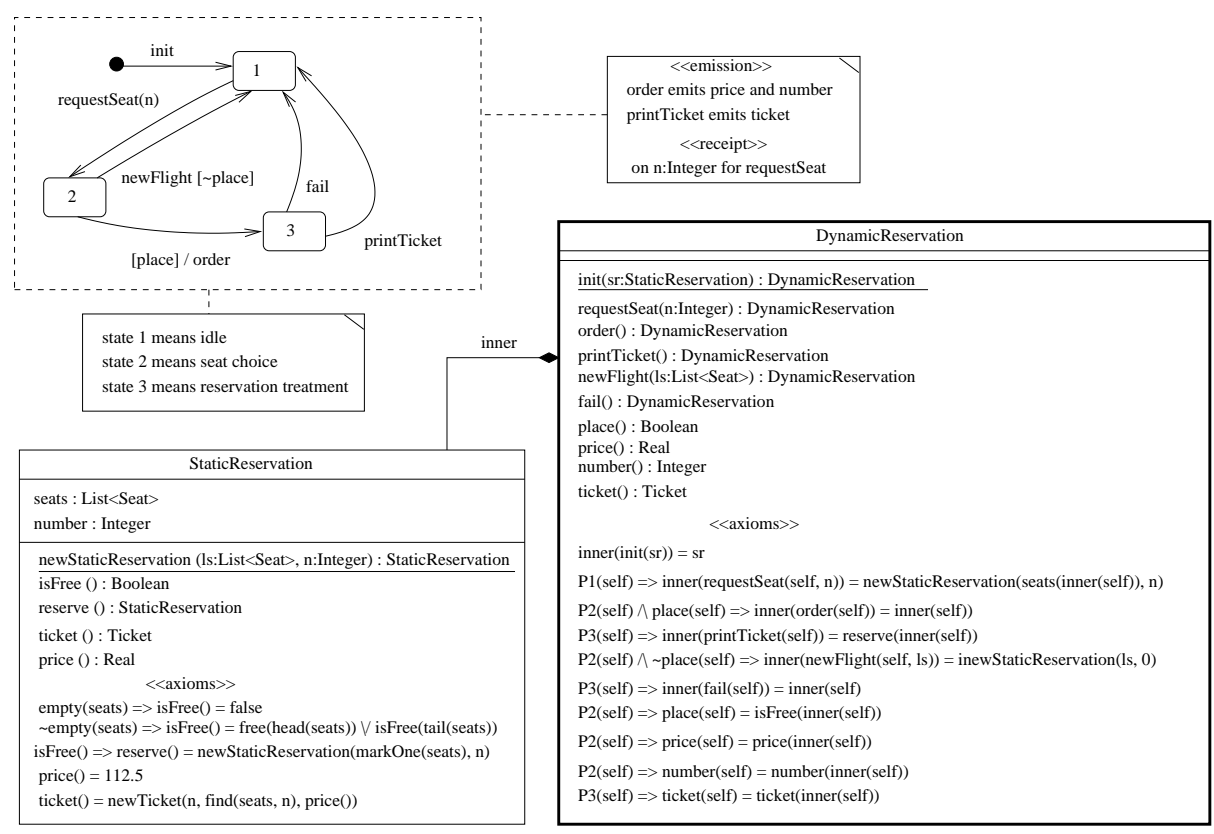

Fig. 3. The Seat Reservation Component 


\subsection{Some Assumptions}

We consider some assumptions and we concentrate only on some essential features ; extensions will complete this approach (see Section 7). We only consider active and static classes, statechart and collaboration diagrams. We only consider side-effect free models, since it simplifies notations, but we may target imperative models with object identity as in [10]. To a sequential or a concurrent process we associate an active class stereotyped process. At this level the difference between thread and process in UML is not relevant. We use algebraic axioms to complete UML diagrams. OCL expressions would be possible but it is rather an operational language than a specification language and it seems to have some lacks [18]. Furthermore we target algebraic languages then OCL would require an additional translation as in [19]. As in the process algebras we consider two kinds of components: sequential (the bank and the seat reservation) or concurrent (the global system).

\subsection{Sequential Component}

For a sequential component, named $\mathrm{T}$, we provide an active class interface (named DynamicT) associated with a static class interface (named StaticT) and a composition link (named inner). The active class as an associated statechart augmented by some notation comments. In the interface description, as usual in UML, the receiver object is implicit.

The static class describes the data used by the active class. It follows the general approach developed in $[9,10]$. There is only one generator corresponding to instantiation and axioms are written in a simple and object oriented way inspired by the formal class model [20].

The statechart describes the dynamic behaviour of the component, the event labelling a transition is assumed to correspond to an operation of the active class. To facilitate the component translation we note emissions and receipts as comments associated to the statecharts. A receipt is associated to a variable of an internal operation of the active class, an emitted value is produced as the result of an operation implicitly associated to a transition. For instance the order action emits two values associated to operations price and number.

For each state state we consider to have a state predicate Pstate. The semantics of operations is described by axioms. The operations of the dynamic part are delegated (if needed) to operations of the static part in a simple way. The axiom:

P2() $\wedge$ exists () $\wedge$ enough () $=>$ inner (success()) = decrease (inner())

means that the success operation, occurring in state 2 and with the guards exists and enough, will decrease the bank account of the inner static instance.

We distinguish basis operations (new) which reach an initial state and internal operations (fail, payment, success) which link two states. Other operations are observers (exists, enough). An operation is total if it is possible in every state, boolean functions (or predicates) are always considered as total ones. The rule to define partiality of other operation is: an operation must be defined in every state where it is needed. For example the exists operation is partial, it is only referenced in guards from state 2 in Figure 2. 


\subsection{Concurrent Component}

The informations to describe a concurrent component are: a class collaboration diagram plus some notations to denote synchronisations and internal communications. There are several ways to express concurrency, synchronisation and communication. Mainly there are: process algebra expression, temporal logic formula or state machine. Our semantics of concurrency is based on the synchronous product of statecharts associated to the components. We may specify asynchronous messages by the way of buffer (we have data in our components) and synchronous messages. Thus we restrict our presentation to synchronisation and communication (the so-called "rendez-vous"). UML uses several notations based on the Harel'statecharts, Petri net notations and messages. We choose a more abstract approach based on the multi-way communication mode of LOTOS. This is obtained by a synchronisation as illustrated in Figure 4. It represents a simple notation for a "rendez-vous". A different way would be to reuse notations of [21] but it will become more difficult to integrate them into our algebraic framework. Another possible way would be to reuse the translation scheme of messages presented in [22]. The synchronisation is completed with notations for emissions and receipts in components and notations for communications in collaboration diagrams.

One example of synchronisation concerns the order action of the seat reservation and the payment action of the bank. To express this synchronisation we use the UML synchronisation bare of the left part of Figure 4. To simplify the figures this synchroni-
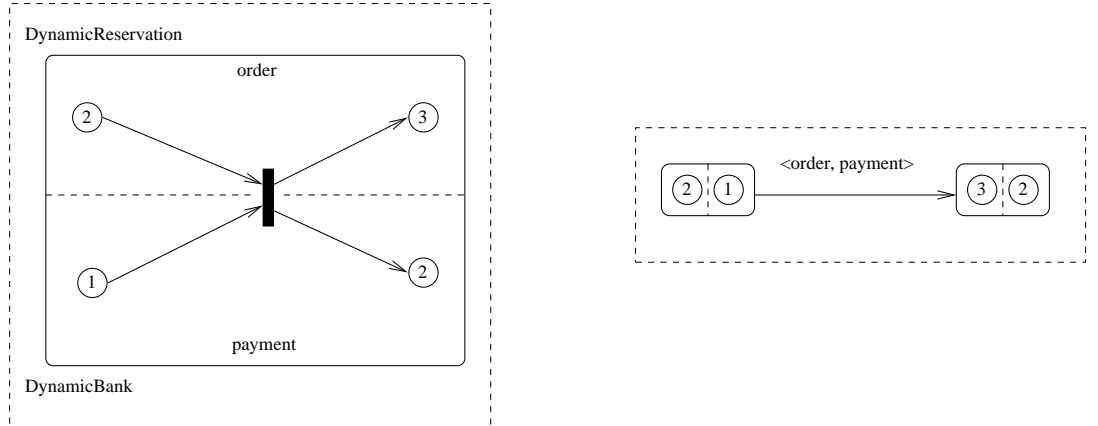

Fig. 4. Synchronisation Representation

sation will be represented as in the right part of Figure 4. However the drawing of such a diagram, in real case study, becomes too complex. Generally the synchronisation rules are based on event names so it is easier to use a class collaboration diagram as in the Figure 5. This gives a better view of the concurrent architecture of the system. Such a kind of concurrency diagram was also suggested in $[6,23]$. We complete the UML diagrams in the following way:

- To use a class collaboration diagram with some simple notations to note synchronisations and internal communications. Internal communications are described in 


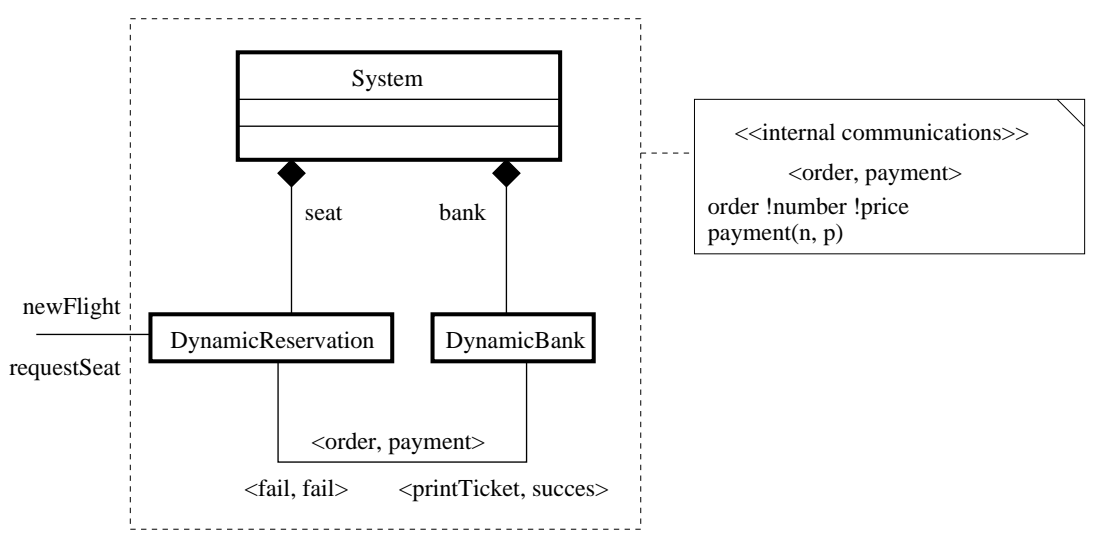

Fig. 5. A Concurrent Architecture for the System

a comment box attached to this diagram. An emission is prefixed by ! and a receipt is a variable parameter. Of course syntactic compatibility is required between emissions and receipts of a synchronisation.

- The interaction order in our collaboration diagram is not really useful because such constraints are well-defined (and in a more concise way) by the statecharts and the synchronisations.

- A last assumption is about the signature for the compound system: parameters of an operation are either provided by internal communications or by external communications.

\section{Translation Rules for Sequential Component}

From the description of Figure 2 we may generate an algebraic specification which represents both the data part and the dynamic part of the diagram. There are two translations: one for the static class and another one for the dynamic class. The dynamic part is translated using several boolean operations denoting definedness of the system, the operation preconditions and the state predicates which code the statechart.

\subsection{Translation of the Static Class}

1. We generate an algebraic specification of an abstract data type with sort name StaticT and which imports all the data types occurring in the class interface (at least we need the data types associated to the attributes of the class).

2. We generate the signature and the axioms of the static class. The signature collects the profiles declared in the static class plus selectors corresponding to the attributes and an instantiation generator. If we have $\left\{a t t_{i}: \mathrm{T}_{i}\right\}_{1 \leq i \leq n}$ as attributes we generate the following instantiation generator:

newstaticT $: \mathrm{T}_{1} \ldots \mathrm{T}_{n} \longrightarrow$ StaticT and the $n$ associated selectors: att $_{i}: \mathrm{T}_{i} \longrightarrow$ StaticT. 
3. We generate axioms for the selectors in the following way: att $_{i}$ (newStaticT $\left.\left(\mathrm{X}_{1}, \ldots, \mathrm{x}_{n}\right)\right)=\mathrm{x}_{i}$.

4. We translate the actual axioms described in the static class:

(a) adding a newStaticT $\left(\mathrm{X}_{1}, \ldots, \mathrm{X}_{n}\right)$ in first place of the left-hand conclusion term which denotes the receiver object.

(b) replacing each att ${ }_{i}$ by the corresponding $X_{i}$ variable in the condition and in the right-hand side conclusion term.

5. Last we declare variables needed in the axioms and the newStaticT as a generator.

The generated algebraic specification for the StaticBank class has been viewed in Figure 1.

\subsection{Translation of the Dynamic Class}

This translation starts from the statechart and the active class.

1. We generate an algebraic specification with the same sort than the class name (named DynamicT). A variable $t$ :DynamicT will denote the receiver object.

2. This algebraic specification imports Boolean, StaticT and the data types occurring in the interface of the dynamic class.

3. We translate the class interface into an algebraic signature, and we add the following operation:

(a) A newDynamict : Statict $\rightarrow$ Dynamict generator.

(b) The inner selector: inner : Dynamict $\rightarrow$ StaticT.

(c) A definedness predicate: Ddynamict : DynamicT $\rightarrow$ Boolean.

(d) One state predicate for each state name:

Pstate : Dynamict $\rightarrow$ Boolean.

(e) For each operation one predicate to denote the precondition and noted Coperation : DynamicT * $\rightarrow$ Boolean, where * represents additional data types required by the operation.

4. We generate axioms for the new operations as following:

(a) For each operation we define the Coperation precondition: for each generator we write Coperation $(t, *)=\exp$, where exp is a disjunction of formulas Pstate $(t) \wedge$ guard $(t, *)$ of the different transitions where the operation is possible.

(b) Axioms for the definedness predicate: for each generator we generate DdynamicT $(g(t, *))$ = DdynamicT $(t) \wedge$ Coperation $(t, *)$.

(c) For each state we define axioms for the state predicates in the following manner. For each generator we write Pstate $\left(g\left(t,{ }^{\star}\right)\right)=\exp$, where exp is the disjunction of the conditions to reach state from a transition labelled by operation $g$.

5. Each line axiom in the dynamic class is translated as for the static class (clause 4 of Section 4.1) into the algebraic specification.

6. We defined variables occurring in the axioms and the set of generator is the set of internal operations. 
A part of the algebraic specification for the DynamicBank is:

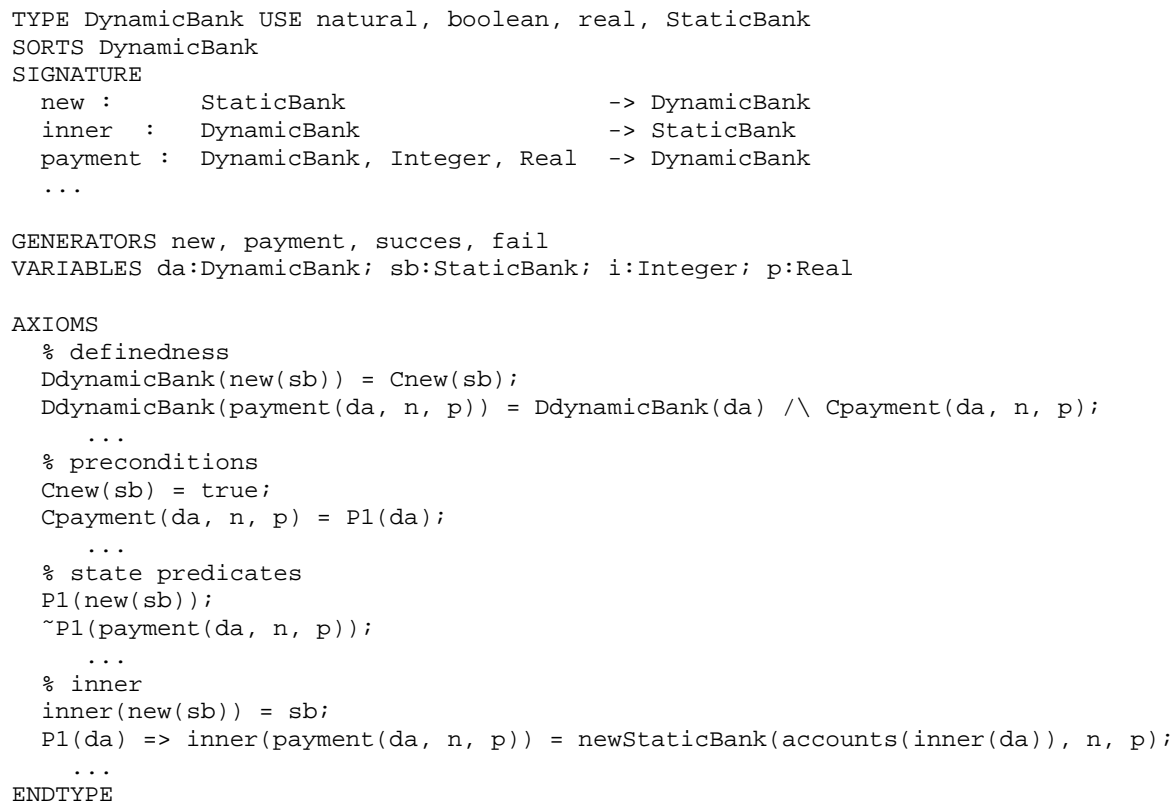

\section{Translation Rules for Concurrent Component}

From the collaboration diagram in Figure 5, we extract the components, the synchronisations and the communications. From that and the component statecharts we generate the synchronous product corresponding to the global dynamic behaviour of the compound system. The principles come from [17,24]. Each state of the product is a compound state which has two inner states corresponding to the component states. The transitions of the product are also compound in the way depicted in Figure 4. There are several possible synchronisation rules (as in LOTOS or CCS for example). Here we choose the same as in LOTOS, but we may change it because it is only a parameter of the product. To take into account the fact that a component may act asynchronously, we use a special nil transition noted - . The resulting statechart product is depicted in the Figure 6. Then from these informations:

- The state machine product is automatically translated into an algebraic specification.

- If the class has proper operations and axioms, they are translated in a similar way than in the sequential case.

The translation of the state machine product is done as follows:

1. The signature contains a newSystem generator with profile newsystem : DynamicReservation, DynamicBank -> Systemand the associated seat and bank selectors. 


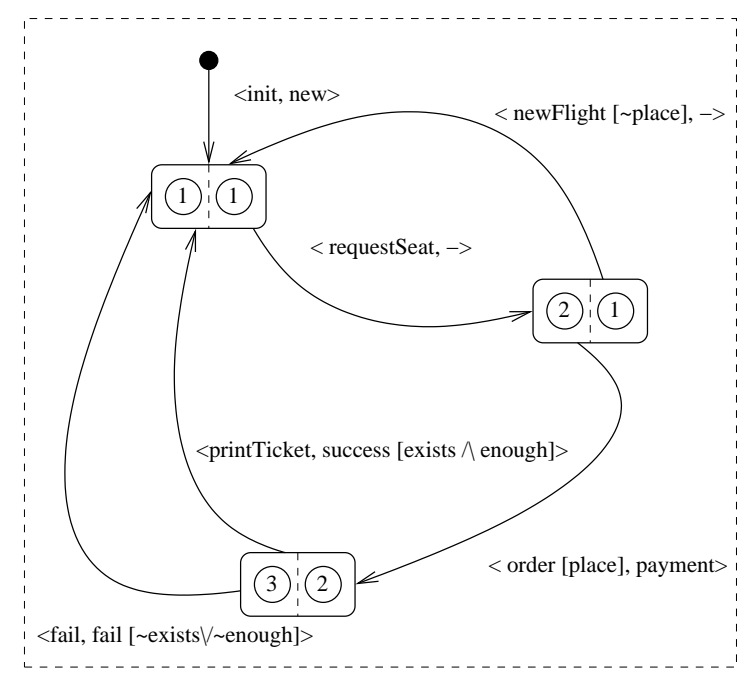

Fig. 6. The Synchronous Product for the System

2. For each kind of transition of the product we associate a label which denotes a generator. Here we choose request Seat for <request Seat, ->, transaction for <order, payment>, deliverfor<printTicket, success $>$, newFlight for <newFlight, $->$ and fail for <fail, fail>. The profile of these operations is obtained by a merging of the component operation profiles coping with component types and internal communications. For example the merging of order : DynamicReservation -> DynamicReservation and payment : DynamicBank Integer Real $->$ DynamicBank gives transaction : System $->$ System

since there are two internal communications (price and number) and the System type results from the composition of DynamicReservation and DynamicBank. Note that variables for external communications are not removed (for instance request Seat has profile System Integer $->$ System).

3. As for the sequential case we add the definedness predicate, the state predicates and the preconditions. The axioms of the definedness, the preconditions and the state predicates are computed in the same way than for the sequential case.

4. Axioms for the selectors are defined with the same principles than for the definition of the inner axioms (see Section 4.2) but taking care of asynchronous or synchronous activities and communications between the components.

\section{The Translation Tool}

In section 2 we talked about the main steps of our approach, in which we mentioned the advantage of automatic generation of algebraic specification from a specifier point of view. UML diagram is just a notation representation, which differs from different 


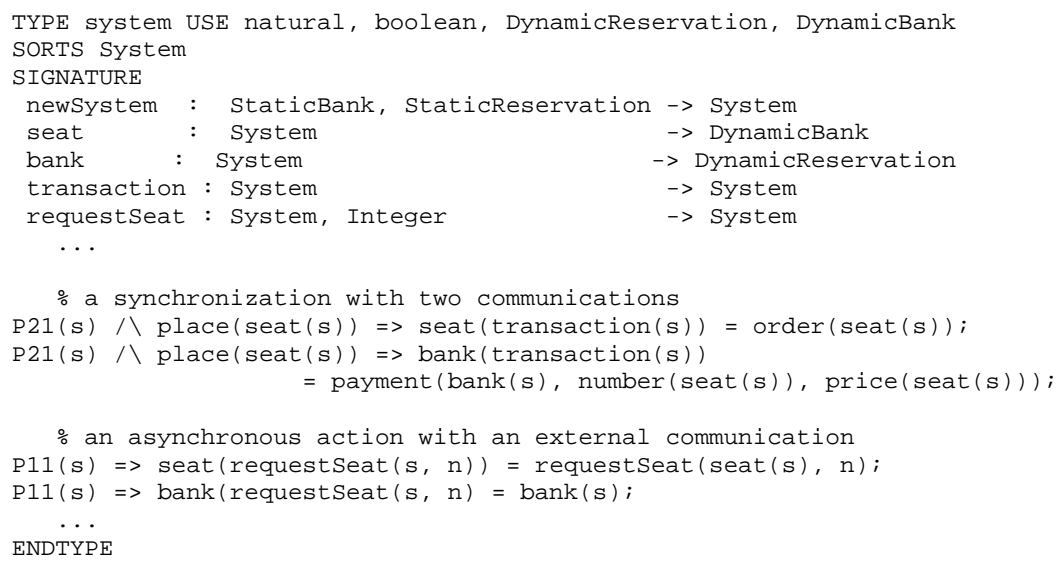

Fig. 7. A Part of the System Specification

CASE tools. So we should find a standard way to represent UML diagram for the free communication between CASE tools and its clarity for analysis purpose. The Rational Rose UML CASE tool is used to design software systems. Here we use it to draw class diagram, statechart diagram and collaboration diagram. Unisys Rose XML tools adds to Rational Rose plug-ins to automatically generate a XMI file from a UML diagram.

\subsection{Driving Technology}

We choose the XMI standard [25] to ensure good interoperability. The main purpose of XMI is to enable easy interchange of metadata between modelling tool (based on the OMG UML) and metadata repositories (OMG MOF based) in distributed heterogeneous environments. XMI integrates three key industry standards: XML - eXtensible Markup Language, UML, and MOF - Meta Object Facility, allowing developers of distributed systems to share object models and other metadata over the Internet. The UML standard defines a rich, object oriented modelling language that is supported by a range of graphical design tools, while XMI allows metadata to be interchanged as streams or files with a standard format based on XML.

The XML4J [26] parser uses both the DTD and the XML document to create DOM tree, which presents the XML document hierarchically. The DOM provides a group of APIs, which allow access to the elements within the tree. Using the DOM APIs, any element within the XML document can be accessed, changed, deleted or added.

\subsection{The Translation Process}

The diagram above (Fig. 8) shows the general working process of our approach. XMI2LP means to translate a XMI file into LP syntax file, and it is developed in Java with the XML4J APIs [26]. We define several classes related to the different parts of the translation. XMI2LP . java is the main file for handling the XMI file translation. CharTool. java implements java utilities for manipulating string and character variable. 


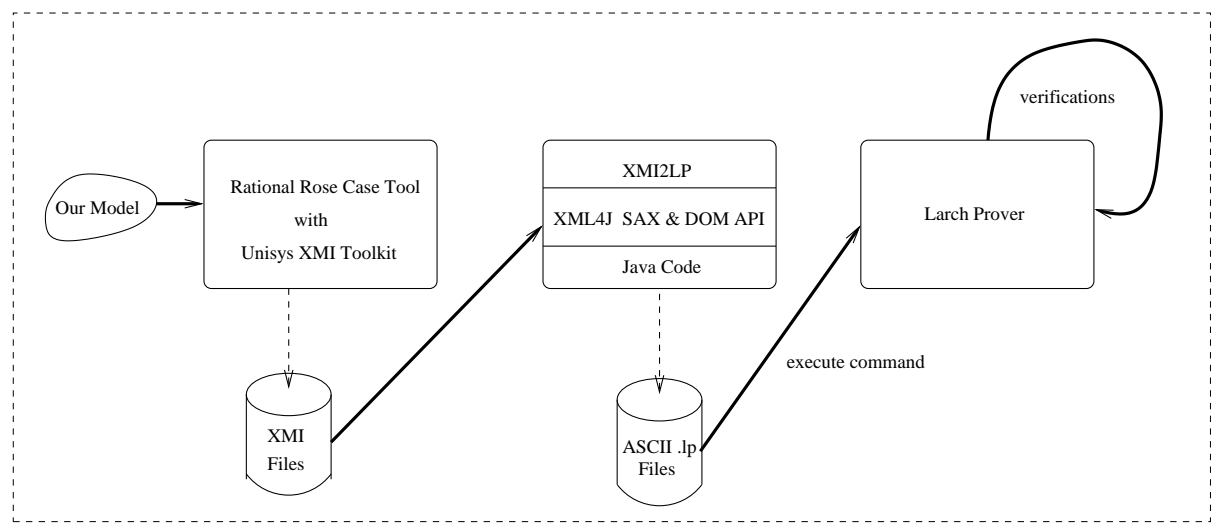

Fig. 8. The XMI2LP process

Transition.java, Operationtype.java, AttributeType.java, Datatype. java, and Association. java are elements used in XMI2LP tool. A representation of the synchronous product of statecharts was done in XML, with two new tags. Then there are also classes to implement the translation of the concurrent case. To day the implementation is complete and we begin to test the tool.

\section{Extensions and Related Works}

We are able to translate only some UML constructions, extensions are needed. There are some previous works which have shown that some restrictions are easy to consider $[18,13]$ then some hypothesis does not really restrict expressiveness. We already studied some extensions like super-state and aggregation state. We have studied inheritance for static classes in [9], for dynamic inheritance a way to solve anomalies may be [27]. More work must be done to cover a large part of UML.

One important milestone in this area is the Rigorous Object Oriented Analysis method of Clark and Moreira [28]. They use LOTOS and E-LOTOS as a target for OMT and more recently for UML. It seems that the idea to model UML use-cases by LOTOS agent [29] is a good approach for these diagrams. Our current approach is related to the application to UML as in [22]. Mainly, they consider active classes, use cases and message sequence charts. Their approach gives a formal semantics to these elements by associating a process to active classes. The formalisation of a use case is done by the notion of interactions and environment of LOTOS. Sequences of messages are translated into process algebraic expressions. Our work is different on several aspects, not only on the fact that we do not yet handle real time constraints. Firstly our approach suggest to reuse a method for LOTOS and SDL to better present UML specifications. Then we consider a restricted (but not too limited) class of systems, nearly open and distributed systems as with LOTOS. Secondly we give a formal specification to static diagrams, active classes, statecharts and collaboration diagrams. We did not cover use cases and both approaches avoid data flow diagrams. We consider that differentiating 
data type and dynamic behaviour is important for a practical point of view in object oriented programming. It is not a good object perspective, as in the ROOA method, to consider static classes as processes. Furthermore we think that abstract description of object behaviours using statechart are essential to reason about UML specifications. Lastly our approach is different on the semantic target: we choose an homogeneous approach based on partial algebraic data types and the use of a theorem prover. Here we use the Larch Prover tool, however our technique has been extended to PVS in [11]. Our approach is able to check inconsistency and also to prove both dynamic properties and data properties as expected in a real framework. The [22] approach is able to test or to simulate some ill-formed situations but it is not really demonstrate how to check problems or prove properties about dynamic behaviour and full data types.

A related approach is [18], it uses labelled transition system and the algebraic language CASL. One very important difference is that we use symbolic transition systems. This avoid many problems of LTS but make proof of temporal properties more difficult, however we described such a way in [5].

Another important work is [30], we used a more constructive approach for the static diagrams, see $[9,10]$. For the dynamic diagram they use pre and post conditions written in OCL; this is an interesting alternative. However the main problem would be concurrency and verification, especially temporal verifications with full data types.

The notion of component we use is rather linked to component in UML-RT [31] than to implementation component of UML, or EJB, Active-X and so on. Thus we need specific notations to define the dynamic interface of a component, its communications with others and concurrency. The present work and UML-RT partly address the same issues: architectural design, dynamic components and reusability. However, UML-RT is at the design level and real time whereas our approach is rather concerned about (formal) specification and logical time issues. There are also some other differences, mainly at the communication level, but the major one is that, to the contrary of UMLRT, we provides a uniform way to specify both data types and behaviours.

\section{Future Work and Conclusion}

The presented approach suggests a method to present UML component specifications based on previous work around LOTOS and algebraic data types. We have shown that a translation of these diagrams is possible and automatic into an algebraic context. This approach is based on a homogeneous semantics for both data types and concurrency, this is a great advantage for verifications. There are other approaches related to this but often they use labelled transition system. One problem is the state and transition explosion problem which ruins the ability to use model-checkers. Our approach is based on symbolic transition system and this has several advantages. It provides abstraction and readability and this remains close to UML statecharts. We also have means to prove temporal properties and in some case it even allows automatic proofs. We have implemented a tool in Java to run this translation. It uses current object oriented technology and XMI standard to achieve portability. The future will extends our approach to additional UML features: state with activities, complex message and activity diagram. We 
also begin to design more complex and real examples and to translate them with our tool thus verifications will become possible.

\section{References}

1. James Rumbaugh, Ivar Jacobson, and Grady Booch. The Unified Modeling Language Reference Manual. Addison-Wesley, 1999.

2. Robert France, Andy Evans, and Kevin Lano. The UML as a formal modeling notation. In Haim Kilov, Bernhard Rumpe, and Ian Simmonds, editors, Proceedings OOPSLA'97 Workshop on Object-oriented Behavioral Semantics, pages 75-81. Technische Universität München, TUM-I9737, 1997.

3. Andy Evans. Making UML precise. In Luis Andrade, Ana Moreira, Akash Deshpande, and Stuart Kent, editors, Proceedings of the OOPSLA'98 Workshop on Formalizing UML. Why? How?, 1998.

4. Pascal Poizat, Christine Choppy, and Jean-Claude Royer. Concurrency and Data Types: a Specification Method. An Example with LOTOS. In J. Fiadero, editor, Recent Trends in Algebraic Development Techniques, Selected Papers of the 13th Workshop on Algebraic Development Techniques, WADT'98, volume 1589 of Lecture Notes in Computer Science, pages 276-291. Springer-Verlag, 1999.

5. Jean-Claude Royer. Formal Specification and Temporal Proof Techniques for Mixed Systems. In Proceedings of the 15th IPDPS 2001 Symposium, FMPPTA, San Francisco, USA, 2001. IEEE Computer Society.

6. Christine Choppy, Pascal Poizat, and Jean-Claude Royer. Specification of mixed systems in KORRIGAN with the support of a UML-inspired graphical notation. In Heinrich Hussmann, editor, Fundamental Approaches to Software Engineering. 4th International Conference, FASE 2001 Held as Part of the Joint European Conferences on Theory and Practice of Software, ETAPS 2001 Genova, Italy, April 2-6. 2001 Proceedings, volume 2029 of LNCS, pages 124-139. Springer, 2001.

7. Stephan Garland and John Guttag. An overview of LP, the Larch Prover. In Proc. of the 3rd International Conference on Rewriting Techniques and Applications, volume 355 of Lecture Notes in Computer Science. Springer-Verlag, 1989.

8. S. Owre, J. M. Rushby, and N. Shankar. PVS: A prototype verification system. In Deepak Kapur, editor, 11th International Conference on Automated Deduction (CADE), volume 607 of Lecture Notes in Artificial Intelligence, pages 748-752. Springer-Verlag, 1992.

9. Pascal André, Annya Romanczuk, Jean-Claude Royer, and Aline Vasconcelos. An Algebraic View of UML Class Diagrams. In H. Sahraoui C. Dony, editor, Acte de la conférence LMO'2000, pages 261-276, 2000. ISBN 2-6462-0093-7.

10. Pascal André, Annya Romanczuk, Jean-Claude Royer, and Aline Vasconcelos. Checking the Consistency of UML Class Diagrams Using Larch Prover. In T. Clark, editor, Proceedings of the third Rigorous Object-Oriented Methods Workshop, BCS eWics, ISBN: 1-902505-38-7, 2000. http://www.ewic.org.uk/ewic/workshop/view.cfm/ROOM2000.

11. Michel Allemand and Jean-Claude Royer. Mixed Formal Specification with PVS. In Proceedings of the 15th IPDPS 2002 Symposium, FMPPTA. IEEE Computer Society, 2002.

12. Jean-Claude Royer. Temporal Logic Verifications for UML: the Vending Machine Example. In Proceedings of the fourth Rigorous Object-Oriented Methods Workshop, 2002.

13. Kevin Lano and Juan Bicarregui. Semantics and transformations for UML models. In PierreAlain Muller and Jean Bézivin, editors, Proceedings of UML'98 International Workshop, Mulhouse, France, June 3 - 4, 1998, pages 97-106. ESSAIM, Mulhouse, France, 1998. 
14. Bertrand Meyer. Object-Oriented Software Construction, 2nd Ed. Prentice-Hall, Englewood Cliffs, NJ 07632, USA, second edition, 1997.

15. E. Astesiano, B.Krieg-Bruckner, and H.-J. Kreowski Eds., editors. Algebraic Foundations of System Specification. IFIP State-of-the-Art Reports. Springer Verlag, 1999. ISBN 3-54063772-9.

16. Martin Wirsing. Algebraic Specification, volume B of Handbook of Theoretical Computer Science, chapter 13, pages 675-788. Elsevier, 1990. J. Van Leeuwen, Editor.

17. André Arnold. Finite Transition Systems. International Series in Computer Science. PrenticeHall, 1994. ISBN 0-13-092990-5.

18. Gianna Reggio, Egidio Astesiano, Christine Choppy, and Heinrich Hussmann. Analysing UML active classes and associated state machines - A lightweight formal approach. In Tom Maibaum, editor, Proc. Fundamental Approaches to Software Engineering (FASE 2000), Berlin, Germany, volume 1783 of LNCS. Springer, 2000.

19. A. Hamie, J. Howse, and S. Kent. Interpreting the Object Constraint Language. In Proceedings of Asia Pacific Conference in Software Engineering. IEEE Press, January 1998.

20. Jean-Claude Royer. An Operational Approach to the Semantics of Classes: Application to Type Checking. Programming and Computer Software, 27(3), 2002. ISSN 0361-7688.

21. Bogumila Hnatkowska and Huzar Zbigniew. Extending the UML with a Multicast Synchronisation. In T. Clark, editor, ROOM'2000: third workshop on Rigorous Object-Oriented Methods, BCS eWics, ISBN: 1-902505-38-7, 2000. http://www.ewic.org.uk/ewic/workshop/view.cfm/ROOM2000.

22. R. Clark and A. Moreira. Use of E-LOTOS in adding formality to UML. Journal of Universal Computer Science, 6(11):1071-1087, 2000.

23. Michael J. McLaughlin and Alan Moore. Real-time extensions to UML. Dr. Dobb's Journal of Software Tools, 23(12):82, 84, 86-93, December 1998.

24. Christine Choppy, Pascal Poizat, and Jean-Claude Royer. The Korrigan Environment. Journal of Universal Computer Science, 7(1):19-36, 2001. Special issue: Tools for System Design and Verification, ISSN: 0948-6968.

25. Unisys Corp. et al. XML Metadata Interchange (XMI), October 1998. ftp: //ftp.omg.org/pub/docs/ad/98-10-05.pdf.

26. IBM. XML4J. Technical report, 1998. http://www.alphaworks.ibm.com/tech/xml 4 j.

27. José Meseguer. Solving the inheritance anomaly in concurrent object-oriented programming. In Oscar Nierstrasz, editor, Proceedings ECOOP'93, LNCS 707, pages 220-246, Kaiserslautern, Germany, July 1993. Springer-Verlag.

28. A. M. D. Moreira and R. G. Clark. Combining Object-Oriented Analysis and Formal Description Techniques. In M. Tokoro and R. Pareschi, editors, Proceedings of ECOOP'94, Bologna, Italy, Lecture Notes in Computer Science 821, pages 344-364. Springer-Verlag, Berlin, 1994.

29. Robert G. Clark and Ana M. D. Moreira. Formal Specifications of User Requirements. Automated Software Engineering: An International Journal, 6(3):217-232, July 1999.

30. A. Hamie, J. Howse, and S. Kent. Modular Semantics for Object-Oriented Models. In Proceedings of Northern Formal Methods Workshop, eWics Series. Springer Verlag, August 1998.

31. Bran Selic and Jim Rumbaugh. Using UML for Modeling Complex Real-Time Systems. Technical report, Rational Software Corp., 1998. 